\title{
Escalas de Depresión, Ansiedad y Estrés (DASS - 21): Validación de la Versión Abreviada en Estudiantes Universitarios Chilenos
}

\author{
Depression Anxiety Stress Scales (DASS - 21): \\ Validation of the Abbreviated Version in Chilean University Students
}

\author{
Zayra Antúnez \\ Universidad Austral de Chile \\ Eugenia V. Vinet \\ Universidad de La Frontera, Chile. \\ (Rec: 24 de mayo de 2011 / Acep: 14 de Diciembre de 2011)
}

\begin{abstract}
Resumen
Debido a la alta tasa de trastornos ansiosos, depresivos y estrés en universitarios, es necesario contar con un instrumento estandarizado en esta población, que permita detectar estas patologías para intervenir oportunamente. Por ello, el objetivo de este trabajo fue determinar las características psicométricas de las Escalas Abreviadas de Depresión, Ansiedad y Estrés (DASS - 21) en universitarios chilenos, lo cual contempló la validación del mismo. La muestra estuvo constituida por 484 estudiantes de la Universidad Austral de Chile, seleccionados mediante un muestreo probabilístico por conglomerados, utilizando un diseño correlacional transversal. La validez de constructo se verificó mediante análisis factorial exploratorio, el que arrojó una estructura de tres factores, explicando el 49,99\% de la varianza total. La validez concurrente y divergente fue verificada con el BAI, BDI - II y SCL - 90 - R. Mediante Alpha de Cronbach se constató la adecuada confiabilidad y consistencia interna del DASS -21 .
\end{abstract}

Palabras clave: DASS - 21, validación, universitarios.

\begin{abstract}
The high rate of anxiety disorders, depression and stress detected in Chilean university students requires a standardized instrument of diagnosis, enabling timely intervention for these disturbances. The aim of this study was to determine the psychometric properties of the Abbreviated Scales of Depression, Anxiety and Stress (DASS-21) for Chilean university students, thus validating the scale. The sample consisted of 484 students of Universidad Austral de Chile selected through random sampling using a cross-sectional correlation design. Construct validity was verified by exploratory factor analysis which yielded a three-factor structure, explaining $49.99 \%$ of total variance. Concurrent and divergent validity was verified by the BAI, BDI-II and SCL-90. Adequate reliability and internal consistency of DASS-21 was found using Cronbach's Alpha. Keywords: DASS - 21, validation, university students.
\end{abstract}

\section{Introducción}

Conceptualmente la depresión y la ansiedad son muy distintas, pero en la práctica clínica e investigación tienden a sobreponerse (Lovibond \& Lovibond, 1995a), ya que por lo general aparecen simultáneamente (Sanderson, DiNardo, Rapee y Barlow, 1990). Por esto, las medidas tradicionales no han podido distinguir bien entre la ansiedad y la depresión (Antony, Bieling, Cox, Enns y Swinson, 1998), siendo creadas para este propósito las Escalas de Depresión, Ansiedad y
Estrés (DASS) (Lovibond \& Lovibond, 1995b). Estas escalas en un principio estaban orientadas a medir solo depresión y ansiedad, no obstante, durante el desarrollo del instrumento surgió una nueva escala, que fue llamada "estrés" (Crawford \& Henry, 2003), la cual mediría un conjunto de síntomas que pueden ser diferenciados de la depresión y la ansiedad, como un estado de activación y tensión persistente.

El DASS quedó constituido por tres escalas (Lovibond \& Lovibond, 1995a) que miden por separado, depresión,

Correspondencia: Mg. Zayra Antúnez, Centro de Salud Universitario, Universidad Austral de Chile. Av. Los Laureles 095, Isla Teja, Valdivia - Chile.

Email: zayraantunez@uach.cl 
ansiedad y estrés. No obstante, los autores plantean que la discriminación perfecta entre medidas de auto - informes de ansiedad y depresión parece imposible (Lovibond \& Lovibond, 1995b), ya que las correlaciones entre las escalas del DASS no se deberían únicamente a la carga de los factores, sino más bien a la continuidad natural entre los tres síndromes y por ende, a la correlación esperable entre los factores, que puede surgir debido a que existen causas comunes subyacentes a la depresión, la ansiedad y al estrés (Lovibond \& Lovibond, 1995b).

Por esto, varios síntomas que pueden considerarse tradicionalmente como parte de alguno de estos tres síndromes, no están representados en las escalas del DASS, ya que no parecen ser específicos de uno u otro síndrome. De este modo, Lovibond \& Lovibond (1995a) conceptualizaron la depresión como un trastorno compuesto por síntomas típicamente asociados con estado de ánimo disfórico (como la tristeza, desesperanza, autodepreciación e inutilidad). Descriptivamente, este síndrome es caracterizado principalmente por una pérdida de la autoestima y el incentivo, asociada a una baja probabilidad de lograr las metas vitales significativas para la persona, entre otros. La ansiedad es descrita en torno a los síntomas físicos de excitación, ataques de pánico, tensión muscular y miedo (temblor o desmayo), destacando los vínculos entre un estado prolongado de ansiedad y una respuesta aguda de miedo; también en esta escala se aborda la ansiedad situacional (Lovibond \& Lovibond, 1995a), entendida como uno de los componentes afectivos del proceso de estrés que en conjunto con otras emociones, como la rabia y el miedo, pueden surgir cuando el individuo no consigue dar respuesta a los estímulos dados (Chaves y Cade, 2004). El estrés es descrito como cierta tendencia a reaccionar a los acontecimientos estresantes con síntomas tales como tensión, irritabilidad y activación persistente, con un bajo umbral para ser perturbado o frustrado (Lovibond $\&$ Lovibond, 1995a), sugiriéndose que hay una continuidad natural entre los síndromes evaluados por la escala de ansiedad y estrés, siendo el punto de división entre los dos un tanto arbitrario (Lovibond \& Lovibond, 1995a).

Considerando que la depresión y la ansiedad representan dimensiones y no categorías, en el desarrollo del DASS se emplearon predominantemente muestras no - clínicas, en las cuales pruebas preliminares arrojaron que el DASS poseía adecuada validez convergente y discriminante (Lovibond \& Lovibond, 1995a). Hasta la fecha, algunos estudios han probado directamente la validez de constructo del DASS, tanto en muestras clínicas como en muestras no clínicas, en jóvenes y adultos (Antony et al., 1998; Brown, Chorpita, Korotitsch \& Barlow, 1997; Clara, Cox \& Enns, 2001; Lovibond \& Lovibond, 1995a). Este último estudio (Lovibond \& Lovibond, 1995a) se realizó en una muestra de estudiantes universitarios con una edad promedio de 21 años $(n=717)$ y reveló, mediante análisis de componentes principales, tres factores correlacionados que representaron una alta proporción de la varianza $(41,3 \%)$. Además, se utilizó análisis factorial confirmatorio para comparar cuantitativamente el ajuste de un modelo de un solo factor, un modelo de dos factores (en el que la depresión es un factor, y la ansiedad y el estrés componen el otro) y un modelo de tres factores que corresponden a las tres escalas del DASS. Se encontró que el modelo de tres factores presenta un significativo mejor ajuste que el modelo de uno y dos factores. En este estudio también se administró el Inventario de Depresión de Beck (BDI) (Beck \& Steer, 1987) y el Inventario de Ansiedad de Beck (BAI) (Beck \& Steer, 1990), encontrándose que el BAI y la escala de ansiedad del DASS están muy correlacionados $(r=0,81)$, así como también el BDI y la escala de depresión de DASS $(r=0,74)$.

Posteriormente, también se desarrolló una versión abreviada del instrumento (DASS - 21) para situaciones en que se necesite realizar una aplicación más breve. Esta última ha sido traducida y validada en población hispana (Daza, Novy, Stanley y Averill, 2002) y han sido analizadas su estructura factorial y propiedades psicométricas, encontrándose una estructura de tres factores mediante análisis factorial confirmatorio (Clara et al., 2001) y análisis factorial exploratorio (Antony et al., 1998). En otros estudios, donde se ha verificado la validez concurrente del DASS - 21, se han encontrado altas correlaciones entre escalas (Apóstolo, Mendes y Azeredo, 2006; Mitchell, Burns \& Dorstyn, 2008). En población hispana, se ha encontrado que el DASS - 21 muestra un ajuste aceptable para un modelo de tres factores y correlaciones importantes entre escalas (Daza et al., 2002). En el estudio realizado por Bados, Solanas y Andrés (2005) en 365 universitarios no consultantes, se encontró una validez divergente y convergente satisfactoria, con correlaciones altas y significativas entre constructos similares. El análisis factorial confirmatorio reveló que un modelo de tres factores correlacionados presentaba un ajuste aceptable (Bados et al., 2005).

Recientemente, también se realizó un estudio de validación del DASS - 21 en Chile (Román, 2010), en estudiantes de enseñanza media, con una edad promedio de 15 años. En este estudio, se utilizó además el BAI y el BDI - II para verificar la validez convergente y discriminante, obteniéndose correlaciones significativas entre la escala de Depresión del DASS -21 y el BDI $(r=0,71)$, y entre la escala de Ansiedad del DASS -21 y el BAI $(r=0,76)$. Asimismo, la escala de Estrés del DASS - 21 se relacionó significativamente tanto con el BAI $(r=0,64)$, como con el BDI $(r=0,57)$. A su vez, mediante análisis factorial confirmatorio se encontró que un modelo de tres factores modificado presentó rangos aceptables, con correlaciones importantes entre factores $(r$ $=0,70$ entre depresión y ansiedad; $r=0,72$ entre depresión y estrés; y $r=0,86$ entre ansiedad y estrés).

En conjunto, las investigaciones previas indican que el DASS - 21 presenta una sólida consistencia interna y proporciona una adecuada distinción entre ansiedad y 
depresión, en relación con otras medidas existentes. Estos estudios también sugieren que la comorbilidad que se da en la práctica clínica podría tener su base en el origen común de los tres síndromes (depresión, ansiedad y estrés) (Brown et al., 1997; Lovibond \& Lovibond, 1995a) y tiene implicancias en el manejo de los tratamientos, ya que aumenta la complejidad clínica, reduce la efectividad de tratamientos habituales y empeora el pronóstico. Por esto, la capacidad de medir por separado estos tres estados puede ser de gran utilidad para los investigadores y clínicos que se ocupan de aclarar el origen de una perturbación emocional, como parte de la tarea más amplia de evaluación clínica, que también permita la planificación de intervenciones para prevenir estos trastornos (Kendall, Hollon, Beck, Hammen \& Ingram, 1987).

En este sentido, la prevención es fundamental en la población universitaria, sobre todo si se considera la particular vulnerabilidad que presentan estos jóvenes para desarrollar trastornos emocionales (Aracena, Barrientos y Rehbein, 1992) y el aumento de estudiantes universitarios en nuestro país, triplicándose desde el año 1981 al año 2000 (Goic, 2002), e incrementándose en un $76 \%$, entre el año 2000 y el 2008 (Meneses, Rolando, Valenzuela y Vega, 2010). Por ende, también aumentan los estudiantes que presentan algún trastorno psicológico, lo cual se explica además, por las altas tasas de prevalencia de problemas de salud mental en la población general chilena (Araya, Lewis, Rojas y Mann, 2003).

Si bien se ha estudiado la presencia de síntomas emocionales, particularmente de sintomatología ansiosa y, especialmente, depresiva (Cova et al., 2007) en universitarios, ninguna investigación ha intentado estudiar la presencia de ansiedad, depresión y estrés en su conjunto, pues tampoco existen instrumentos adaptados a la realidad de nuestros estudiantes, siendo el objetivo de esta investigación, determinar las características psicométricas (validez de constructo, convergente y discriminante, y confiabilidad por consistencia interna) de una versión chilena del DASS - 21 en estudiantes universitarios.

\section{Método}

\section{Participantes}

La población correspondió a estudiantes de pregrado de la Universidad Austral de Chile, que cursaban entre primer y cuarto año de sus respectivas carreras $(N=8983$ en el año 2008). Los participantes fueron seleccionados mediante un muestreo aleatorio por conglomerados, según carreras y niveles (de $1^{\circ}$ a $4^{\circ}$ año). El tamaño muestral se determinó utilizando la fórmula para estimar un promedio poblacional en una población finita sin reposición, usando un nivel de confianza del 99\% $(z=2,576)$. Los datos concernientes al DASS fueron tomados del estudio de Bados et al., (2005), quedando constituida la muestra final por 484 estudiantes.

La muestra que se obtuvo presentó las siguientes características sociodemográficas: jóvenes no consultantes entre 18 y 28 años ( $M: 20.2, D T: 2.1$ ), siendo un $44.8 \%$ de sexo masculino. La mayoría procedía de zonas urbanas (86.4\%) y un porcentaje menor (12.2\%) de zonas rurales, con un origen familiar predominantemente no mapuche (83.4\%) y en menor porcentaje, con un origen familiar mapuche (16.5\%). La mayoría de ellos era soltero (97\%) y su distribución socioeconómica correspondió principalmente al nivel medio (23\% Medio - Bajo, 28\% Medio y $30 \%$ Medio - Alto).

\section{Diseño}

Se trabajó con un diseño no experimental correlacional, de carácter transversal.

\section{Instrumentos}

Los instrumentos utilizados en orden de aplicación fueron el instrumento en estudio: Escalas de Depresión, Ansiedad y Estrés (DASS - 21), y los instrumentos de validación: Inventario de Ansiedad de Beck (BAI), Inventario de Depresión de Beck, segunda edición (BDI - II) e Inventario de Síntomas de Derogatis Revisado ( $\mathrm{SCL}-90$ - R). Además se utilizó un cuestionario sociodemográfico de construcción ad-hoc que incluyó la Escala ESOMAR (ADIMARK, 2000) para clasificar el nivel socioeconómico de los participantes. A continuación se describe brevemente cada uno de ellos.

Versión chilena abreviada de las Escalas de Depresión, Ansiedad y Estrés (DASS - 21). Esta fue traducida y adaptada en Chile por Vinet, Rehbein, Román y Saiz (2008) y modificada por Román (2010), siendo esta última versión la utilizada. El DASS - 21 cuenta con 21 ítems, con cuatro alternativas de respuesta en formato Likert, las cuales van desde 0 ("No describe nada de lo que me pasó o sentí en la semana") hasta 3 ("Sí, esto me pasó mucho, o casi siempre"). Para responder, la consigna establece indicar en qué medida la frase describe lo que le pasó o sintió la persona durante la última semana. Este instrumento tiene la ventaja de ser una escala de auto reporte, breve, fácil de administrar y responder, siendo su interpretación sencilla. Además, ha presentado adecuadas propiedades psicométricas en estudios anteriores de validación (Antony et al., 1998; Apóstolo et al., 2006; Clara et al., 2001) y un ajuste aceptable a un modelo de tres factores en muestras de habla hispana (Bados et al., 2005; Daza et al., 2002; Román, 2010).

Inventario de Ansiedad de Beck (BAI). Es un instrumento autoaplicable de 21 ítems, desarrollado para estimar ansiedad, discriminando entre ésta y la sintomatología depresiva (Beck \& Steer, 1990). Aunque fue diseñado para ser utilizado en población clínica, Sanz, Navarro y Vázquez (2003) demuestran, en universitarios ibéricos, resultados positivos tanto 
respecto a su consistencia interna (alfa $=0,88)$, como a su capacidad para identificar ansiedad patológica en población no clínica. Asimismo, estudios realizados en nuestro país, tanto con adolescentes (Cova, Melipillán, Valdivia, Bravo y Valenzuela, 2007) como con universitarios (Cova et al., 2007), permiten verificar las propiedades psicométricas del instrumento y la capacidad de discriminar efectivamente entre adolescentes chilenos con ansiedad normal y patológica, al utilizar un punto de corte de 16 .

Inventario de Depresión de Beck, segunda edición (BDI - II). Este instrumento autoaplicable consta de 21 ítems y presenta adecuadas propiedades psicométricas (Beck et al., 1988; Piotrowski, 1996; Sanz et al., 2003). Ha sido utilizado en Chile en adolescentes, demostrando que discrimina efectivamente entre población clínica y no consultante con un puntaje de corte de 19, el cual indica presencia de sintomatología depresiva importante. La consistencia interna $($ alfa $=.91)$ y la estructura factorial observada en Chile son semejantes a las de otros estudios (Cova, Melipillán, Rincón y Valdivia, 2008).

Inventario de Síntomas de Derogatis Revisado (SCL - 90 - R). Instrumento desarrollado por Derogatis (1975, 1994) para evaluar el grado de malestar psicológico actual que experimenta una persona. Permite caracterizar la sintomatología del evaluado en un perfil compuesto por nueve dimensiones primarias de síntomas (Somatización, Obsesiones, Sensitividad, Interpersonal, Depresión, Ansiedad, Hostilidad, Ansiedad Fóbica, Ideación Paranoide y Psicoticismo) y tres índices globales de psicopatología (Índice de Gravedad Global, Índice de Malestar Positivo y Total de Síntomas Positivos). Ha sido adaptado, validado y estandarizado en estudiantes universitarios chilenos no consultantes por Gempp y Avendaño (2008), exhibiendo un desempeño psicométrico aceptable. Es autoadministrado, fácil de responder y corregir, y consta de 90 preguntas en formato Likert, las que pueden responderse en aproximadamente 15 minutos.

El cuestionario sociodemográfico permitió recoger antecedentes relevantes y del contexto académico de los participantes, como género, carrera, curso, edad, nivel socioeconómico. Este último se midió con el Método de Evaluación del Nivel Socioeconómico ESOMAR (ADIMARK, 2000), que permite establecer el nivel socioeconómico familiar a partir del nivel educacional y de la categoría ocupacional del principal sostenedor del hogar.

\section{Procedimiento}

Se solicitó la aprobación a los Comités de Ética de la Universidad de La Frontera y de la Universidad Austral de Chile. Luego se envió una carta a los directores de cada carrera solicitando su colaboración y autorización para la administración de los cuestionarios en periodo de clases. Previo a la aplicación, se solicitó a los estudiantes colaboración en la investigación a través de su participación voluntaria, la cual fue formalizada mediante la firma de un consentimiento informado.

\section{Resultados}

Los datos fueron analizados mediante el programa estadístico SPSS, versión 11.5. A continuación se exponen los resultados más relevantes:

\section{Análisis Factorial}

Con el objetivo de verificar la validez de constructo del DASS - 21 y de observar la distribución de los ítems en los factores, se realizó un análisis factorial exploratorio. En este se usó el método de extracción de componentes principales y rotación Oblimin con Kaiser. En primera instancia, el análisis factorial exploratorio se realizó con una solución libre, el cual arrojó cuatro factores, no obstante, el cuarto factor se caracterizó, en su mayoría, por cargas factoriales menores a .20, dificultando su interpretación teórica.

En segunda instancia se realizó un análisis factorial exploratorio con una solución forzada a tres factores, el que arrojó valores mayores a .30 en los ítems. La viabilidad de este análisis fue avalada por un índice KMO de .909 y por la Prueba de Esfericidad de Barlett, con un valor $p$ significativo de .000. Mediante este análisis se logró determinar la estructura de los tres factores que expresan las escalas que componen el DASS - 21, los cuales serían: Factor 1: Estrés, Factor 2: Depresión y Factor 3: Ansiedad. Estos factores se presentan a continuación en el gráfico de sedimentación, con los valores propios (autovalores), donde se observa que el punto de inflexión se encuentra en el factor 3 , indicando una estructura de 3 factores que explican el $49.99 \%$ de la varianza total.

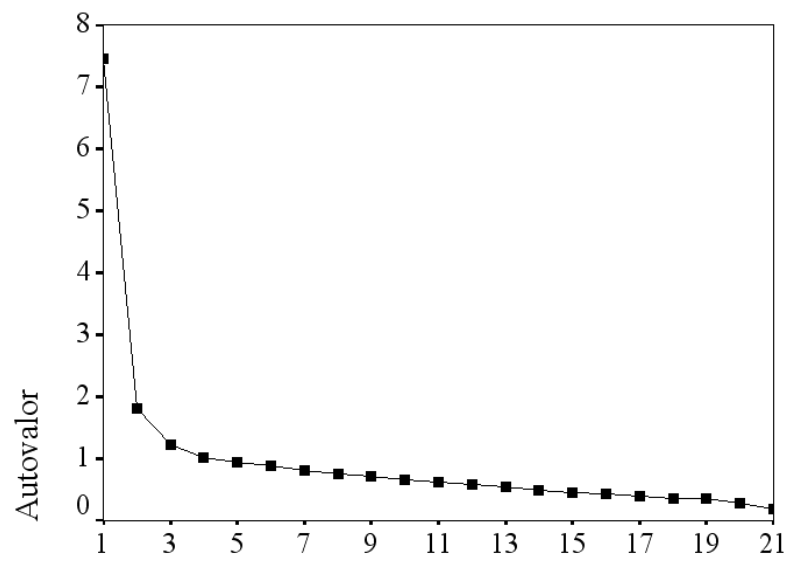

Número de factor

Figura 1: Gráfico de sedimentación. 
Las cargas factoriales de cada ítem en los factores correspondientes y los porcentajes de varianza contenida por cada factor extraído, así como los autovalores, se presentan a continuación en la Tabla 1. Se observa que el factor Estrés explicaría un $35.5 \%$ de la varianza, el factor Depresión un $8.62 \%$ de la varianza y el factor Ansiedad explicaría un $5.87 \%$ de la varianza. Como puede observarse en la Tabla 1 , si bien los ítems definidos teóricamente se agrupan del mismo modo empíricamente, hay algunos ítems que presentan carga significativa o relevante, es decir, mayor a .30 en dos factores. Estos serían específicamente el ítem 8: "Sentí que estaba muy nervioso/a”, el cual pertenece al factor Estrés y también pesa en el factor Ansiedad. Y un conjunto de ítems que perteneciendo al factor de Depresión, pesan también en

Tabla 1: Matriz de configuración con factores rotados y autovalores.

\begin{tabular}{|c|c|c|c|c|}
\hline \multirow[b]{2}{*}{$\begin{array}{l}\text { Ítems } \\
\text { DASS - } 21\end{array}$} & \multirow[b]{2}{*}{$\begin{array}{c}\text { Factor al que } \\
\text { pertenece }\end{array}$} & \multicolumn{3}{|c|}{ Factores } \\
\hline & & $\begin{array}{c}\text { F1: } \\
\text { Estrés }\end{array}$ & $\begin{array}{c}\text { F2: } \\
\text { Depresión }\end{array}$ & $\begin{array}{c}\text { F3: } \\
\text { Ansiedad }\end{array}$ \\
\hline Ítem 18 & F1: Estrés & .72 & & \\
\hline Ítem 6 & F1: Estrés & .72 & & \\
\hline Ítem 11 & F1: Estrés & .68 & & \\
\hline Ítem 1 & F1: Estrés & .66 & & \\
\hline Ítem 12 & F1: Estrés & .65 & & \\
\hline Ítem 8 & F1: Estrés & .50 & & .43 \\
\hline Ítem 14 & F1: Estrés & .38 & & \\
\hline Ítem 5 & F2: Depresión & .58 & .36 & \\
\hline Ítem 10 & F2: Depresión & & .88 & \\
\hline Ítem 21 & F2: Depresión & & .83 & \\
\hline Ítem 17 & F2: Depresión & & .67 & \\
\hline Ítem 16 & F2: Depresión & .41 & .53 & \\
\hline Ítem 3 & F2: Depresión & .40 & .51 & \\
\hline Ítem 13 & F2: Depresión & .40 & .47 & \\
\hline Ítem 19 & F3: Ansiedad & & & .76 \\
\hline Ítem 4 & F3: Ansiedad & & & .62 \\
\hline Ítem 20 & F3: Ansiedad & & & .58 \\
\hline Ítem 7 & F3: Ansiedad & & & .56 \\
\hline Ítem 9 & F3: Ansiedad & & & .40 \\
\hline Ítem 15 & F3: Ansiedad & & & .30 \\
\hline Ítem 2 & F3: Ansiedad & & & .27 \\
\hline Autovalor & & 7.455 & 1.811 & 1.233 \\
\hline $\begin{array}{l}\text { Porcentaje d } \\
\text { explicada }\end{array}$ & varianza & 35.499 & 8.622 & 5.872 \\
\hline $\begin{array}{l}\text { Porcentaje d } \\
\text { acumulado }\end{array}$ & varianza & 35.499 & 44.121 & 49.993 \\
\hline
\end{tabular}

Nota. Los ítemes se presentan agrupados por factor y en orden según peso factorial. el factor Estrés. Estos son los ítems 5: "Se me hizo dificil motivarme para hacer cosas", 16: "Fui incapaz de entusiasmarme con nada", 3: "No podía sentir nada positivo" y 13 "Me sentí triste y deprimido/a". Finalmente, en el factor de Ansiedad se agrupan tanto teórica como empíricamente los ítems que lo componen, siendo solo el ítem 2: "Me di cuenta que tenía la boca seca", el que presenta un peso factorial bajo (.27), considerando que una carga factorial desde .30 es aceptable (Gutiérrez, 2010).

En cuanto a la correlación que se dio entre los factores, la mayor correlación se presentó entre los factores de estrés y ansiedad $(r=.46)$, seguida por la correlación entre estrés y depresión $(r=.37)$ y por último, entre depresión y ansiedad $(r=.24)$.

\section{Análisis Correlacional}

Se realizaron análisis correlacionales con $r$ de Pearson entre el DASS - 21 y los otros instrumentos utilizados, con el fin de determinar la validez concurrente y discriminante de las escalas del DASS - 21. Los resultados indican que la escala de depresión del DASS - 21 correlaciona en mayor medida con el Inventario de Depresión de Beck (BDI), con un valor $r$ de .70, correlacionando también con la escala de depresión del SCL $-90-\mathrm{R}(r=.63)$ y con el Índice de Gravedad Total del SCL $-90-\mathrm{R}$ con un $r=.62$, mientras que la asociación con las otras escalas es menor.

La escala de ansiedad del DASS - 21 correlaciona altamente tanto con el Inventario de Ansiedad de Beck (BAI) con un $r$ de .78, como con la escala de ansiedad del

Tabla 2: Correlación Escalas DASS - 21 con otros Instrumentos.

\begin{tabular}{lccc}
\hline \multicolumn{1}{c}{ Escala } & $\begin{array}{c}\text { DASS - } \\
\text { Depresión }\end{array}$ & $\begin{array}{c}\text { DASS - } \\
\text { Ansiedad }\end{array}$ & $\begin{array}{c}\text { DASS- } \\
\text { Estrés }\end{array}$ \\
\hline BAI Total & .57 & .78 & .71 \\
BDI - II Total & .70 & .63 & .70 \\
SCL - Ansiedad & .47 & .68 & .68 \\
SCL - Depresión & .63 & .53 & .58 \\
SCL - Índice Gravedad & .62 & .66 & .70 \\
Total & & & \\
SCL - Índice Malestar & .55 & .52 & .59 \\
Positivo & & & .64 \\
SCL - Total Síntomas & .56 & .63 & \\
Positivos & & & \\
\hline
\end{tabular}

Nota. Todas las correlaciones son significativas al nivel .001 (bilateral). 
SCL $-90-\mathrm{R}(r=.68)$. Tal como sucede con la escala de depresión del DASS - 21, también esta escala correlaciona con el Índice de Gravedad Total del SCL - 90 - R con un $r=.66 \mathrm{y}$ en menor magnitud con las otras escalas.

La escala de estrés del DASS - 21 correlaciona significativamente tanto con el BAI $(r=.71)$, como con el BDI $(r=.70)$ y el índice de gravedad total del SCL $-90-\mathrm{R}$ $(r=.70)$. En menor medida correlaciona con la escala de ansiedad del SCL $-90-\mathrm{R}(r=.68)$ y con las otras escalas.

\section{Análisis de Confiabilidad DASS - 21}

Las escalas de depresión y estrés presentaron un alfa de .85 y .83 respectivamente, mientras que en la escala de ansiedad se obtuvo un alfa de .73. En conjunto, los ítems que componen el DASS - 21 presentaron un alfa de .91.

Asimismo, el BAI presentó un alfa de .87 y el BDI - II arrojó un índice de consistencia interna alfa de .88. Estos datos respaldan la consistencia interna de ambos instrumentos al ser aplicados en una muestra de universitarios chilenos. En tanto, el SCL - $90-\mathrm{R}$ presentó adecuados índices de consistencia interna en todas sus escalas, siendo solo la escala de Ansiedad Fóbica la que presenta un valor alfa menor a .70 .

\section{Discusión}

Los resultados obtenidos indican que el DASS - 21 sería un instrumento confiable, con un desempeño psicométrico aceptable en población universitaria no consultante chilena, con una adecuada validez de constructo, convergente y discriminante, así como una sólida consistencia interna.

Debido a que uno de los objetivos de la presente investigación fue determinar la validez de constructo, y puesto que este es el primer estudio chileno en que se realiza una aplicación masiva del DASS - 21 en jóvenes universitarios, se consideró el análisis factorial exploratorio como el más pertinente, permitiendo observar el comportamiento psicométrico del instrumento en la muestra utilizada y los posibles influjos de la cultura en los resultados.

De este modo, al realizar el análisis factorial exploratorio, la matriz resultante mostró sólidos índices de factorización (KMO: .909 y Barlett: .000) y una estructura de 3 factores interpretables teóricamente (Estrés, Depresión y Ansiedad), donde los ítems presentaron un valor aceptable (mayor a .30 ), con un único valor menor (.27 en el ítem "Me di cuenta que tenía la boca seca", correspondiente a Ansiedad). Se sugiere que este ítem sea revisado en futuras investigaciones, ya que tal vez en estudiantes chilenos no refleja adecuadamente el constructo medido o es entendido por los jóvenes de diversas formas, no relacionándolo en primera instancia con ansiedad, sino más bien con sed causada, por ejemplo, por consumo excesivo de tabaco o de algún medicamento, por exceso de deporte o simplemente por bajo consumo de líquidos, como lo evidenciaron algunos de ellos, al realizar preguntas sobre este ítem al momento de responder el test.

Si bien la mayoría de los ítems presentó peso factorial en los componentes a los que pertenecían teóricamente, algunos de ellos presentaron carga simultáneamente en dos factores, lo que concuerda con los antecedentes teóricos y empíricos antes mencionados. Esto podría deberse, a las características comunes que los tres trastornos presentan (Lovibond \& Lovibond, 1995b), a la comorbilidad que tiende a presentarse entre la ansiedad, la depresión y el estrés (Costello, Foley \& Angold, 2006) y/o a que el estrés tendría características comunes a la ansiedad y la depresión (Norton, 2007), siendo la ansiedad uno de sus componentes afectivos (Chaves \& Cade, 2004). Esto concuerda específicamente con los resultados obtenidos, donde la mayor correlación se da entre los factores de ansiedad y estrés $(r$ $=.46$ ), coincidiendo además con los resultados obtenidos recientemente por Román (2010) en una muestra de adolescentes chilenos. Asimismo, destaca la alta proporción $(35.5 \%)$ de la varianza que explica el factor de estrés, lo que haría considerar el DASS - 21 como un aporte en la detección de este trastorno en al ámbito universitario. Esto cobra relevancia al considerar la alta prevalencia de este síndrome en esta población (Norton, 2007), que puede precipitar episodios de ansiedad y depresión (Selye, 1952), y que es considerado un estado de afectividad negativa que generaría situaciones de vulneralibidad emocional (Brown et al., 1997).

De esta forma, los resultados arrojados por el análisis factorial exploratorio serían acordes con lo planteado teóricamente y señalado empíricamente por distintos autores y representarían los constructos tal como se dan en la práctica clínica, es decir, con comorbilidad. En síntesis, este análisis permitió determinar el número de factores y la estructura interna del DASS - 21 en la población utilizada.

En cuanto a la validez convergente y divergente, se observaron correlaciones considerables entre escalas del DASS - 21 y los instrumentos utilizados (y escalas de éstos) que miden constructos similares $(r=.63$ para la escala de depresión del DASS y escala de depresión del SCL - 90 - R, y $r=.78$ para la escala de ansiedad del DASS con BAI). A su vez, tanto la menor correlación entre escalas del DASS 21 y escalas de SCL - 90 - R que miden otras dimensiones, como entre el BAI y la escala de depresión del DASS -21 $(r=.57)$, y entre el BDI - II y la escala de ansiedad del DASS - $21(r=.63)$, respaldarían la validez divergente.

Acerca de la confiabilidad de las escalas, son destacables los índices de consistencia interna que presentaron las escalas de Depresión, Ansiedad y Estrés (DASS - 21), evidenciando una adecuada confiabilidad que se reflejó en valores sobre el alfa .73 en sus escalas. Cabe mencionar que los instrumentos utilizados para evaluar la validez convergente y divergente del DASS - 21 (BDI, BAI y SCL -90 - R) presentaron índices de confiabilidad total mayores al 
alfa .87 , lo que avalaría la consistencia y estabilidad de los resultados obtenidos con estos instrumentos.

Se recomienda que estudios posteriores continúen estudiando las propiedades psicométricas de las escalas, especialmente en muestras más amplias de estudiantes y en jóvenes de diferentes zonas del país y de distintos grupos étnicos, con el fin de determinar el nivel de estabilidad y generalidad del DASS - 21 en estudiantes chilenos.

\section{Referencias}

ADIMARK (2000). El Nivel Socio Económico ESOMAR, Manual de Aplicación. ADIMARK. Extraído el 15 de junio de 2010 desde http:/ www.microweb.cl/idm/documentos/ESOMAR.pdf

Antony, M., Bieling P., Cox B., Enns M., \& Swinson R. (1998). Psychometric properties of the 42 - item and 21 - item versions of the Depression Anxiety Stress Scales in clinical groups and a community sample. Psychological Assessment, 10, 176-181.

Apóstolo, J., Mendes, A., y Azeredo, Z. (2006). Adaptación para la lengua portuguesa de la depression, anxiety and stress scale (DASS). Revista Latino - Americana de Enfermagem, 14, 863 - 871.

Aracena, M., Barrientos, P., y Rehbein, L. (1992). Prevalencia de trastornos emocionales en estudiantes de la Universidad de la Frontera. Revista Frontera, 11, 19-27.

Araya, R., Lewis, G., Rojas, G., y Mann, A. (2003). Patient knows best - detection of common mental disorders in Santiago, Chile: Cross sectional study. British Medical Journal, 322, 79 - 81 .

Bados, A., Solanas, A., y Andrés, R. (2005). Psychometric properties of the Spanish version of Depression, Anxiety and Stress Scales (DASS). Psicothema, 17, 679-683.

Beck, A., \& Steer, R. (1987). Manual for the revised Beck Depression Inventory. San Antonio, United States: The Psychological Corporation.

Beck, A. \& Steer, R. (1990). Beck anxiety inventory manual. San Antonio, United States: The Psychological Corporation.

Brown, T., Chorpita B., Korotitsch, W., \& Barlow, D. (1997). Psychometric properties of the depression anxiety stress scales (DASS) in clinical samples. Behaviour Research and Therapy, 35, 79-89.

Chaves, E., y Cade, N. (2004). Anxiety effects on blood pressure of women with hypertension. Revista Latino - Americana de Enfermagem, 12, $162-7$.

Clara, I., Cox, B., \& Enns, M. (2001). Confirmatory factor analysis of the Depression Anxiety Stress Scales in depressed and anxious patients. Journal of Psychopathology and Behavioral Assessment, 23, 61 - 67.

Costello, E., Foley, D., \& Angold, A. (2006). 10 - Year research update review: The epidemiology of child and adolescent psychiatric disorders: II. Developmental Epidemiology. American Academy of Child \& Adolescent Psychiatry, 45, 8 - 25.

Cova, F., Alvial, W., Aro, M., Bonifetti, A., Hernández, M., y Rodríguez, C. (2007). Problemas de salud mental en estudiantes de la Universidad de Concepción. Terapia Psicológica, 25, 105 - 112.

Cova, F., Melipillán, R., Valdivia, M., Bravo, E., y Valenzuela, B. (2007). Sintomatología depresiva y ansiosa en estudiantes de enseñanza media. Revista Chilena de Pediatría, 78, 151 - 159.

Cova, F., Melipillán, R., Rincón P., y Valdivia, M. (2008). Propiedades psicométricas de Inventario de Depresión de Beck - II en adolescentes chilenos. Terapia Psicológica, 26, 59- 69.
Crawford, J., \& Henry J. (2003). The Depression Anxiety Stress Scales (DASS): Normative data and latent structure in a large non - clinical sample. British Journal of Clinical Psychology, 42, 111 - 131.

Daza, P., Novy, D., Stanley, M., y Averill, P. (2002). The depression anxiety stress scale - 21: Spanish translation and validation with a hispanic sample. Journal of Psychopathology and Behavioral Assessment, 24, $195-205$.

Derogatis, L. (1975). The SCL - 90 - R. Baltimore. United States: Clinical Psychometric Research.

Derogatis, L. (1994). SCL-90-R. Administration, scoring and procedures manual. Minneapolis, United States: National Computer Systems.

Gempp, R., y Avendaño, C. (2008). Datos normativos y propiedades psicométricas del SCL $-90-\mathrm{R}$ en estudiantes universitarios chilenos. Terapia Psicológica, 26, $39-58$.

Goic, A. (2002). Proliferación de escuelas de medicina en Latinoamérica: Causas y consecuencias. Revista Médica de Chile, 130, 917 - 924.

Gutiérrez, R. (2010). Análisis factorial. Extraído el 26 mayo de 2010 desde http://www.ugr.es/ ramongs/sociologia/tema10 factorial.pdf.

Kendall, P., Hollon, S., Beck, A., Hammen, C., \& Ingram, R. (1987). Issues and recommendations regarding use of the Beck Depression Inventory. Cognitive Therapy and Research, 11, 289-299.

Lovibond, P., \& Lovibond, S. (1995a). The structure of negative emotional states: comparison of the depression anxiety stress scales (DASS) with the Beck depression and anxiety inventories. Behaviour Research and Therapy, 33, $335-343$.

Lovibond, S., \& Lovibond P. (1995b). Manual for the depression anxiety stress scales. Sydney, Australia: Psychology Foundation of Australia.

Meneses, F., Rolando, R., Valenzuela, M., y Vega, M. (2010). Ingreso a la educación superior: La experiencia de la cohorte de egreso 2005. División de Educación Superior, Ministerio de Educación de Chile. Extraído el 22 de junio de 2010 desde http://www.divesup.cl/images/archivos/ Publicaciones/ingreso\%20a\%201a\%20educacin\%20superior.pdf.

Mitchell, M., Burns, N., y Dorstyn, D. (2008). Screening for depression and anxiety in spinal cord injury with DASS-21. Spinal Cord, 46, 547-551.

Norton, J. (2007). Depression anxiety and stress scales (DASS - 21): Psychometric analysis across four racial groups. Anxiety, Stress \& Coping, 20, $253-265$.

Piotrowski, C. (1996). Use of the beck depression inventory in clinical practice. Psychological Reports, 79, $873-874$.

Román, M. (2010). Validación de la versión abreviada de las escalas de depresión, ansiedad y estrés (DASS - 21) en adolescentes estudiantes de enseñanza media de la comuna de Temuco. Tesis de Magíster no publicada, Universidad de La Frontera, Temuco, Chile.

Sanderson, W., Di Nardo, P., Rapee, R., y Barlow, D. (1990). Syndrome comorbidity in patients diagnosed with a DSMIII - R anxiety disorder. Journal of Abnormal Psychology, 99, 308-312.

Sanz, J., Navarro, M., y Vázquez, C. (2003). Adaptación española del Inventario para la Depresión de Beck-II (BDI-II): Propiedades psicométricas en estudiantes universitarios. Análisis y Modificación de Conducta, 29, $239-288$.

Selye, H. (1952). The story of the adaptation syndrome. Montreal, Canadá: Montreal University Press.

Vinet, E., Rehbein, L., Román F., y Saiz, J. (2008). Escalas abreviadas de depresión, ansiedad y estrés (dass - 21). Versión chilena traducida y adaptada. Documento no publicado, Universidad de La Frontera, Temuco, Chile. 
